\title{
QTL analysis of percentage of grains with chalkiness in Japonica rice (Oryza sativa)
}

\author{
X. Liu ${ }^{1,2}$, Y. Wang ${ }^{2}$ and S.W. Wang ${ }^{3}$ \\ ${ }^{1}$ Key Laboratory of Food Nutrition and Safety, \\ Tianjin University of Science and Technology, Ministry of Education, \\ Tianjin, China \\ ${ }^{2}$ Department of Plant Biology and Ecology, College of Life Sciences, \\ Nankai University, Tianjin, China \\ ${ }^{3}$ Department of Agronomy, Tianjin Agriculture College, Tianjin, China \\ Corresponding author: X. Liu \\ E-mail: liuxia831930@yahoo.com.cn
}

Genet. Mol. Res. 11 (1): 717-724 (2012)

Received April 27, 2011

Accepted December 15, 2011

Published March 22, 2012

DOI http://dx.doi.org/10.4238/2012.March.22.1

\begin{abstract}
Appearance quality of rice grains is a major problem for rice production in many areas of the world. We conducted a molecular marker-based genetic analysis of percentage of grains with chalkiness (PGWC), which is a determining factor for appearance quality; it strongly affects milling, eating and cooking quality. An $\mathrm{F}_{8}$ recombinant inbred line population, which consists of 261 lines derived from a cross between Koshihikari (Japonica) and C602 (Japonica), was used for QTL mapping. Three QTLs related to PGWC were detected on chromosomes 5, 8 and 10, together explaining $50.8 \%$ of the genetic variation. The 'Koshihikari' alleles qJPGC-5, qJPGC-8 and the 'C602' alleles at qJPGC-10 were associated with reduced PGWC. The QTL contributions to phenotypic variance were 18.2, 9.6 and $25 \%$, respectively. These QTL markers for PGWC could be used for developing improved varieties.
\end{abstract}

Key words: Rice; Recombinant inbred line population; Quantitative trait loci; Percentage of grain with chalkiness 


\section{INTRODUCTION}

Chalkiness is a major concern in rice (Oryza sativa L.) breeding because it is one of the key factors in determining quality and price. Percentage of grains with chalkiness (PGWC) is one of the main indices of rice-determining appearance quality. For improvement of milling, eating, and cooking quality, the endosperm of rice varieties should be free of chalkiness, since chalky grains have a lower density of starch granules compared to vitreous ones and are therefore more prone to breakage during milling (Del Rosario et al., 1968). Also, since both longitudinal and transverse cracks occur easily in chalky kernels when the grain is steamed or boiled, chalkiness reduces the palatability of cooked rice (Nagato and Ebata, 1959). Since consumers prefer rice free of opaqueness and strong translucence, breeding for rice grains free of chalkiness is considered to be within market requirements.

Yield and quality are two major subjects for many rice breeding programs but they are both controlled by quantitative trait loci (QTLs) showing continuous phenotypic variation in rice progeny (Yano and Sasaki, 1997). The inheritance of rice grain chalkiness, an endosperm quality trait, can be more complicated because the genetic expression of an endosperm trait in cereal seeds is controlled not only by the triploid endosperm genotype, but also by the diploid maternal genotype and any additional possible cytoplasmic differences (Pooni et al., 1992; Zhu and Weir, 1994; Mo, 1995). It is difficult for breeders to improve rice grain chalkiness using conventional methods, due to the lack of a discrete phenotypic segregation in the progeny. Thus, identification of QTLs involved in grain chalkiness and the elucidation of its genetic control are necessary for the development of marker-assisted selection (MAS) and pyramid breeding strategies aimed at improving breeding efficiency.

The availability of almost unlimited numbers of molecular markers and genetic maps of high resolution in rice provides powerful tools for QTL mapping and gene cloning. Using different mapping populations, some PGWC QTLs were found (Koh et al., 1999; He et al., 1999; Tan et al., 2000; Li et al., 2004; Wan et al., 2005; Huang, 2006). Whitebelly endosperm trait has been fine mapped on chromosome 7, where 13 predicted genes were confirmed (Zhou et al., 2009). Two of these genes, OSPPDKB and starch synthase IIIa (SSIIIa), were found to have pleiotropic effects on white-core endosperm in rice (Kang et al., 2005; Fujita et al., 2007; Ryoo et al., 2007). The former encodes pyruvate orthophosphate dikinase, which contributes to the control of carbon flow into starch and lipid biosynthesis during grain filling (Kang et al., 2005), while SSIIIa plays an important role in the elongation of amylopectin chains (Fujita et al., 2007). However, the coherence is very low in different background materials for PGWC QTLs. Since all the above studies were conducted in Indica/Japonica or Indica/Wild population background, the Japonica/Japonica population of PGWC QTLs was not evaluated. However, PGWC QTLs are critical for MAS breeding in a Japonica genetic background.

Fine mapping of stable PGWC in different backgrounds is important for gene cloning of chalkiness. PGWC QTLs in a Japonica/Japonica population and their genetic regulation were analyzed in the present study to elucidate the regulation and basis of the development of rice grain chalkiness in Japonica subspecies. 


\section{MATERIAL AND METHODS}

\section{Genetic materials}

Koshihikari is a typical Japonica variety, in which PGWC is 9\%. C602 is a Japonica variety but has some Indica origin, and it has a PGWC of 59\%.

Recombination inbred line (RILs) populations were used for PGWC genetic and QTL analyses; they were produced by crossing Koshihikari (Japonica) and C602 (Japonica). In total, $261 \mathrm{~F}_{8}$ descendants of Koshihikari/C602 were generated through single-seed descent.

\section{Field experiment design and implementation}

Koshihikari, C602 and RILs of Koshihikari/C602 were grown in the summer of 2007 (rice growing season) on the experimental farms of Tianjin Academy of Agricultural Sciences, Tianjin, China. After drying, the RIL grains were stored at room temperature for 3 months, and then dehulled and scored for PGWC and other traits. Each plot consisted of four rows of 10 plants each, arranged in a randomized block design with three replicates.

\section{Scanning electron microscope (SEM) analysis of rice grains}

For SEM observation, kernels were broken transversely and the pieces were mounted onto 12-mm aluminum stubs, and sputtered with gold on a BioRad Polaron sputter coater. Samples were viewed with an S-2500 Hitachi SEM and examined using SEM at an accelerating voltage of $20 \mathrm{kV}$. Diameters of starch granules were estimated on the basis of the scale bar provided on the captured image.

\section{Trait measurement}

PGWC was evaluated according to He et al. (1999) and NSPRC (1999). To separate chalky from vitreous grains, three replicates of 100 grains per entry were assessed visually. The mean percentage of chalky grains represented the PGWC score for that line.

\section{DNA preparation and polymerase chain reaction (PCR) protocol}

Rice total DNA was extracted using the CTAB method (Doyle, 1991). Simple sequence repeats (SSRs) were used for PGWC QTL analysis, where 236 SSR markers distributed at certain genetic distances across all 12 chromosomes (Gramene release; http://www. gramene.org/microsat/ssr.html; accessed November 17, 2005). PCR was performed following the techniques of Chen et al. (1997). PCR products were separated on a 3\% agarose gel.

\section{Linkage map construction and QTL surveys}

For the whole genome survey, 236 SSRs were employed in the assay of the Koshihikari/C602 RILs, and the molecular marker linkage maps were constructed using MAPMAKER/EXP 3.0 (Lander et al., 1987; Lincoln et al., 1992). 
Interval mapping was carried out to scan the whole genome for putative QTLs controlling the PGWC traits using Windows QTL Cartographer 2.5 (http://statgen.ncsu.edu/ qtlcart/) with an LOD threshold of 3.0. If two or more QTLs were detected in nearby regions, the QTL with the largest effect was fixed, and the rest of the genome was rescanned to confirm the existence of other QTLs. When a QTL was detected in an interval exceeding 20 $\mathrm{cM}$ in length in the linkage map, the existence of the QTL was confirmed using composite interval mapping (Zeng, 1994) as well as one-way analysis of variance using genotypes of the adjacent markers as the groups.

\section{RESULTS}

\section{Phenotype of chalky rice and genetic analysis of PGWC}

The chalky appearance of rice is associated with the development of numerous air spaces between starch granules and the resulting change in light reflection (Tashiro and Wardlaw, 1991). Figure 1A and B show the morphology of vitreous and chalky rice in Koshihikari and $\mathrm{C} 602$, respectively. SEM analysis showed that starch granules of the chalky part were round and loosely packed in $\mathrm{C} 602$, which were very different from the densely packed, polyhedral starch granules of the Koshihikari transparent part (Figure 1C and D). This was somewhat consistent with the result of Lisle et al. (2000).

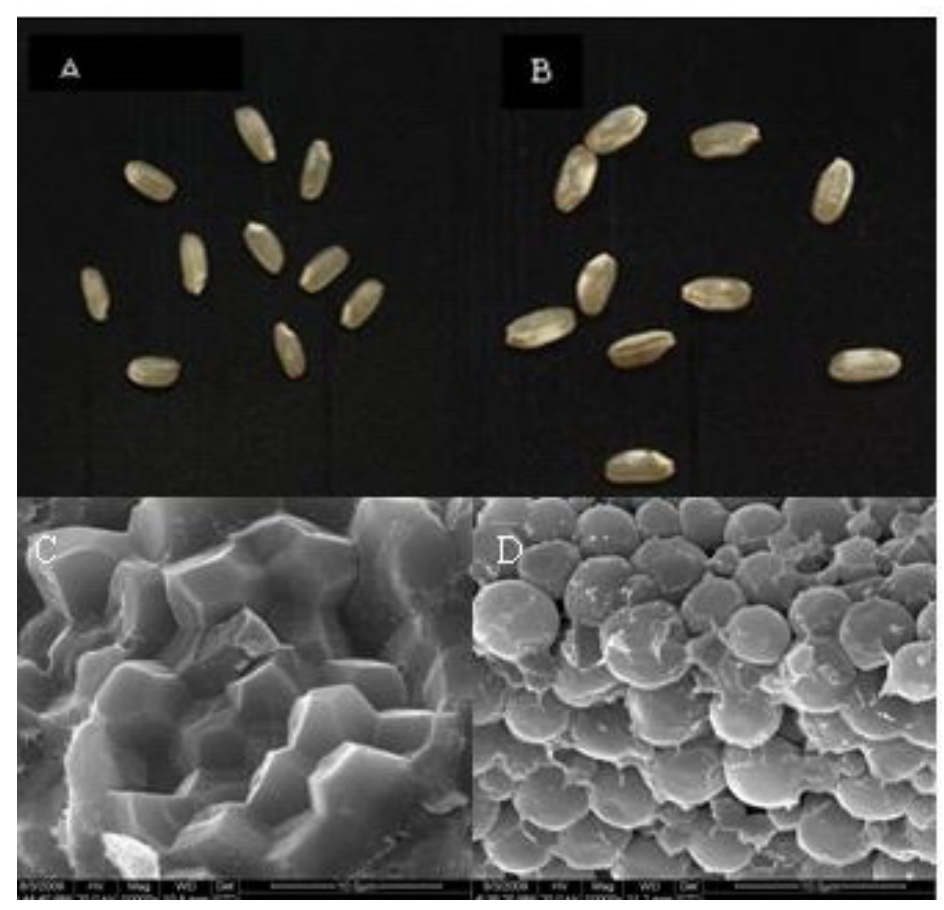

Figure 1. Starch granule morphology and structure of Koshihikari (A), C602 (B). SEM images of starch granules in the mature endosperm of Koshihikari, transparent part (C), C602 chalkiness, grain chalky part (D). Bar represents $10 \mu \mathrm{m}$ in $\mathrm{C}$ and $\mathrm{D}$. 
There were significant differences in PGWC between Koshihikari and C602; their PGWC values were 56 and 9\%, respectively. The distributions of PGWC in Koshihikari ( $\mathrm{Ja}$ ponica)/C602 (Japonica) RIL populations are shown in Figure 2. The PGWC of the Koshihikari/C602 population ranged from 0 to $100 \%$, mainly around 0 to $60 \%$ or so, showing continuous variation. The distribution mode of chalkiness showed a continuous variation and very different with an apparent bimodal and unimodal distribution in the RIL population (Figure 2). In the Koshihikari/C602 RILs, a certain number of RIL PGWC values segregated transgressively to their female parent, indicating that PGWC is a quantitative trait. At the same time, triploid endosperm, cytoplasmic and diploid maternal genetic effects may all be of importance for PGWC and chalkiness area (Shi et al., 2002).

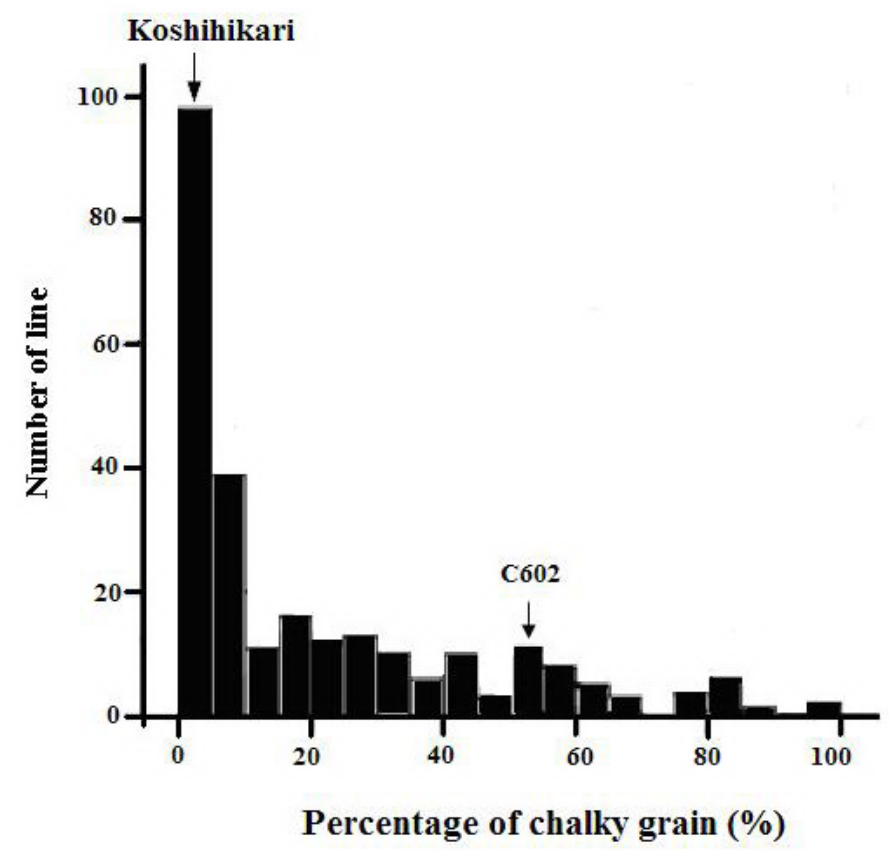

Figure 2. Frequency distributions of percentage of grains with chalkiness (PGWC) in Koshihikari/C602 recombinant inbred line population and its parents' PGWC. The plants were grown in Tianjin in 2007.

\section{QTLs for PGWC in Koshihikari/C602 (Japonica/Japonica population)}

Analysis of the Koshihikari/C602 $\mathrm{F}_{8}$ RIL population revealed three QTLs for PGWC of rice, located on chromosomes 5, 8 and 10, respectively (Table 1, Figure 3 ) in the Japonica/ Japonica RIL population. The QTL qJPGC-5, which was located in the interval on chromosome 5, can explain $25 \%$ of the phenotypic variance. The effects of qJPGC- 8 and qJPGC-10 were much smaller, their variances were 18.2 and $9.6 \%$, respectively. The total phenotypic variation explained by the three putative QTLs was $50.8 \%$. Of the three QTLs, two QTL alleles from C602 contributed to an increase in PGWC, and only one QTL allele from Koshihikari contributed to an increase in PGWC, which was located on chromosome 10. 


Table 1. Putative quantitative trait loci (QTLs) of rice percentage of chalky grain trait estimated for Koshihikari/
C602 recombinant inbred lines.
\begin{tabular}{lclcrr}
\hline QTL & Chromosome & Marker interval & Variation explained (\%) & LOD & Add \\
\hline qJPGC-8 & 8 & RM447-RM281 & 18.2 & 6.35 & 20.43 \\
qJPGC-10 & 10 & RM1873-68923-7 & 9.6 & 3.28 & -1.56 \\
qJPGC-5 & 5 & RM289-RM3437 & 25.0 & 8.62 & 9.81 \\
Total & & 50.8 & 17.32 & \\
\hline
\end{tabular}

LOD $=$ logarithm of the odds score.

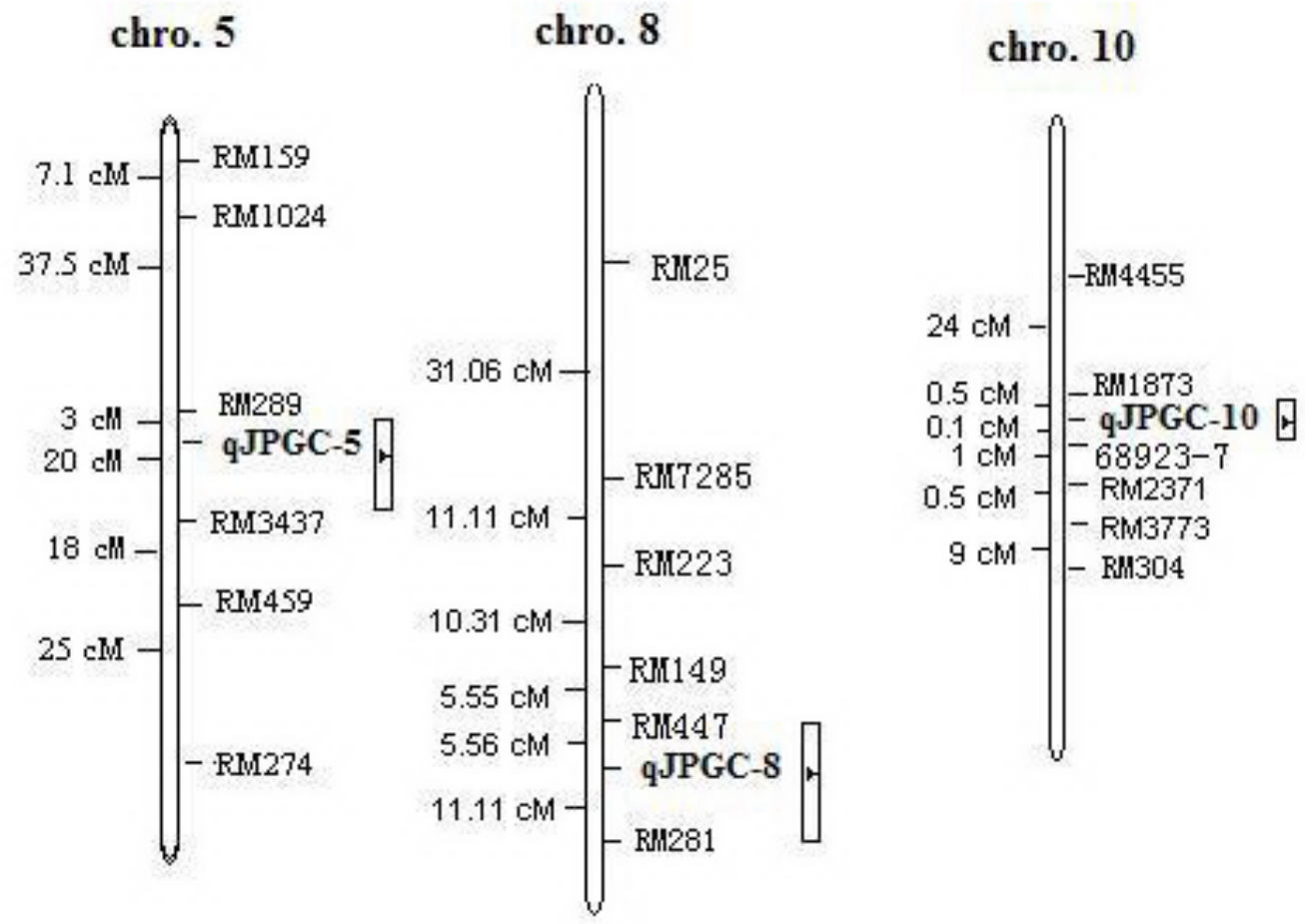

Figure 3. Map locations of the putative quantitative trait loci (QTLs) for percentage of chalky grains detected using Koshihikari/C602 recombinant inbred line populations. The bars indicate the confidence intervals of QTL. The peaks of the QTL interval bar are indicated by black triangles. The scale in Kosambi cM is shown on the left of each chromosome.

Among the three QTLs, qJPGC-10 was mapped on chromosome 10 between SSR markers RM1873 and 68923-7, and the DNA sequence length between them was about 880 $\mathrm{kb}$. Based on this result, we designed the PCR primer pair 68923-jcp10. Using the 68923jcp 10 primer, PCR was performed using DNA samples from the two parents, individual plants with more than $80 \%$ PGWC and individual plants with $0 \%$ PGWC, respectively (Figure 4). The results showed that the fragment amplified by the $68923-j c p 10$ primer was closely associated with PGWC; high-PGWC individuals had the positive band and low-PGWC individuals lacked this band (Figure 4). Thus, the SSR marker 68923-jcp10 could be used in MAS of qJPGC-10 and breeding by design for high/low PGWC in rice. 


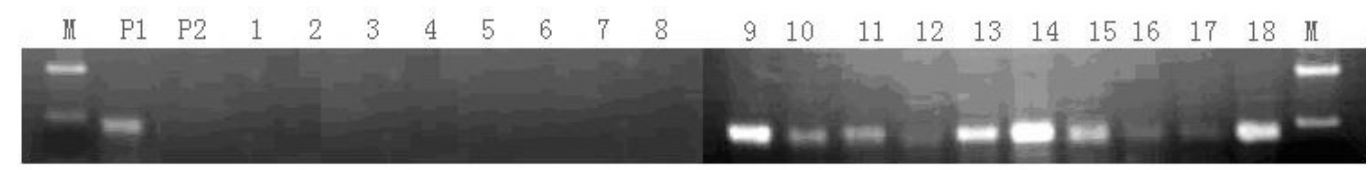

Figure 4. Progeny analysis with 68923-jcp10. Lane $M=\mathrm{DL} 2000$ marker; lane $P 1=$ Koshihikari; lane P2 = C602; lanes $1-8=$ recombinant inbred lines (RILs) that have $0 \%$ chalky grains; lanes $9-18=$ RILs that have more than $80 \%$ chalky grains.

\section{DISCUSSION}

Grain chalkiness is a complicated physiological process in the grain filling phase and was not consistent in previous PGWC QTL detection by different population. Gene identification of different germplasm of PGWC is important for elucidating the molecular mechanism accounting for grain chalkiness. Some QTLs, which can be detected in different populations, may act on general physiological processes; they do not diverge in subspecies evolution. In addition, some different loci may be affected by the genes of specific subspecies or environmental factors. In this study, we detected qJPGC-5, qJPGC-8 and qJPGC-10 in a Japonica background population, which were located on chromosomes 5, 8 and 10, respectively. qJPGC-5 and qJPGC-10 may act on Japonica and Indica backgrounds, because these two QTL were detected between an Indica and Indica population (Tan et al., 2000). But qJPGC-8 can only be found in Japonica background according to the results of known QTL loci of PGWC integration.

The formation of rice chalkiness proves to be a complicated physiological process and tightly related to "source-sink" in rice, dynamics of grain filing, and biosynthesis and accumulation of starch in the endosperm. There are some stable QTLs for rice chalkiness on many rice chromosomes. Of them, three genes controlling rice chalkiness, which have an impact on starch synthesis, starch metabolism, and fruit development, have been cloned. However, the manipulative network and formative mechanisms of rice chalkiness remain unclear. At present in breeding practice, reducing rice chalkiness has become one of the main aims in rice quality breeding.

\section{ACKNOWLEDGMENTS}

Research supported by the National Natural Science Foundation of China Grant (\#31000826), the 2009 46th China Postdoctoral Science Foundation (\#20090460698) and the 3rd Special Foundation China Postdoctoral Science (\#201003303).

\section{REFERENCES}

Chen X, Temnykh S, Xu Y, Cho YG, et al. (1997). Development of a microsatellite framework map providing genomewide coverage in rice (Oryza sativa L.). Theor. Appl. Genet. 95: 553-567.

Del Rosario AR, Briones VP, Vidal AJ and Juliano BO (1968). Composition and endosperm structure of developing and mature rice kernel. Cereal Chem. 45: 225-235.

Doyle JJ (1991). DNA Protocols for Plants-CTAB Total DNA Isolation. In: Molecular Techniques in Taxonomy (Hewitt GM, ed.). Springer, Berlin Heidelberg, New York, 283-293.

Fujita N, Yoshida M, Kondo T, Saito K, et al. (2007). Characterization of SSIIIa-deficient mutants of rice: the function of 
SSIII $a$ and pleiotropic effects by SSIIIa deficiency in the rice endosperm. Plant Physiol. 144: 2009-2023.

He P, Li SG, Qian Q, Ma YQ, et al. (1999). Genetic analysis of rice grain quality. Theor. Appl. Genet. 98: 502-508.

Huang JX (2006). Genetic Analysis and QTL Mapping Research of Appearance Quality Traits in Indica Rice. Master's thesis, Xiamen University, Xiamen.

Kang HG, Park S, Matsuoka M and An G (2005). White-core endosperm floury endosperm-4 in rice is generated by knockout mutations in the C-type pyruvate orthophosphate dikinase gene (OSPPDKB). Plant J. 42: 901-911.

Koh HJ, Son YH, Heu MH, Lee HS, et al. (1999). Molecular mapping of a new genic male-sterility gene causing chalky endosperm in rice (Oryza sativa L.). Euphytica 106: 57-62.

Lander ES, Green P, Abrahamson J, Barlaw A, et al. (1987). Mapmarker: an interactive computer package for maps of experimental and nutural populations. Genomics 1: 174-181.

Li J, Xiao J, Grandillo S, Jiang L, et al. (2004). QTL detection for rice grain quality traits using an interspecific backcross population derived from cultivated Asian (O. sativa L.) and African (O. glaberrima S.) rice. Genome 47: 697-704.

Lincoln S, Daly M and Lander ES (1992). Construction Genetic Maps with MAPMARKER/EXP 3.0. Whitehead Institute Technical Report. 2nd edn. Whitehead Institute for Biomedical Research, Cambridge.

Lisle AJ, Martin M and Fitzgerald MA (2000). Chalky and translucent rice grains differ in starch composition and structure and cooking properties. Cereal Chem. 77: 627-632.

Mo HD (1995). Identification of genetic control for endosperm traits in cereals. Acta Genet. Sin. 22: 126-132.

Nagato K and Ebata M (1959). Studies on white-core rice kernel II. On the physical properties of the kernel. Proc. Crop Sci. Soc. Jpn. 28: 46-50.

NSPRC (1999). National Standard of People Republic of China High Quality Paddy, GB/T 17891-1999. Standards Press of China, Zhejiang.

Pooni HS, Kumar I and Khush GS (1992). A comprehensive model for disomically inherited metrical traits expressed in triploid tissues. Heredity 69: 166-174.

Ryoo N, Yu C, Park CS, Baik MY, et al. (2007). Knockout of a starch synthase gene OsSSIIIa/Flo5 causes white-core floury endosperm in rice (Oryza sativa L.). Plant Cell Rep. 26: 1083-1095.

Shi CH, Wu JG, Lou XB, Zhu J, et al. (2002). Genetic analysis of transparency and chalkiness area at different filling stages of rice (Oryza sativa L.). Field Crops Res. 76: 1-9.

Tan YF, Xing YZ, Li JX, Yu SB, et al. (2000). Genetic bases of appearance quality of rice grains in Shanyou 63, an elite rice hybrid. Theor. Appl. Genet. 101: 823-829.

Tashiro T and Wardlaw IF (1991). The effect of high temperature on kernel dimensions and the type and occurrence of kernel damage in rice. Aust. J. Agric. Res. 42: 485-496.

Wan XY, Wan JM, Weng JF, Jiang L, et al. (2005). Stability of QTLs for rice grain dimension and endosperm chalkiness characteristics across eight environments. Theor. Appl. Genet. 110: 1334-1346.

Yano M and Sasaki T (1997). Genetic and molecular dissection of quantitative traits in rice. Plant Mol. Biol. 35: 145-153.

Zeng ZB (1994). Precision mapping of quantitative trait loci. Genetics 136: 1457-1468.

Zhou L, Chen L, Jiang L, Zhang W, et al. (2009). Fine mapping of the grain chalkiness QTL qPGWC-7 in rice (Oryza sativa L.). Theor. Appl. Genet. 118: 581-590.

Zhu J and Weir BS (1994). Analysis of cytoplasmic and maternal effects II. Genetic models for triploid endosperm. Theor. Appl. Genet. 89: 160-166. 